\title{
An Investigation into the unexpected corrosion of nickel alloy vessels with Selectfluor
}

Tao Wang, ${ }^{\dagger *}$ Thomas P. Vickery, ${ }^{\dagger *}$ Donald Bachert, ${ }^{\dagger}$ Rekha Gangam,${ }^{\dagger}$ Eric Margelefsky, ${ }^{\dagger}$ Eric M. Phillips, ${ }^{\dagger}$ Stephen M. Dalby ${ }^{\dagger}$ Wenjun Liu, ${ }^{\ddagger}$ Amanda Peterman, ${ }^{\dagger}$ Kerstin Zawatzky,${ }^{\ddagger}$ Ryan D. Cohen ${ }^{\ddagger}$

$†$ Department of Process Research and Development, Merck \& Co., Inc., Rahway, New Jersey 07065, United States

$\$$ Department of Analytical Research and Development, Merck \& Co., Inc., Rahway, New Jersey 07065, United States

\section{Supporting Information}

\section{Table of Contents}

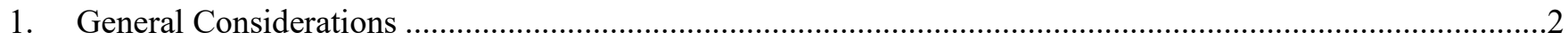

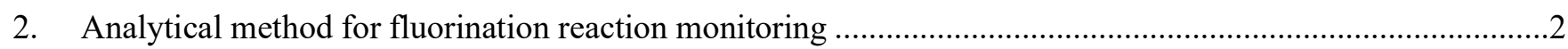

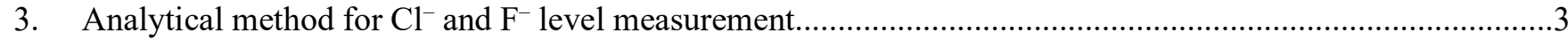

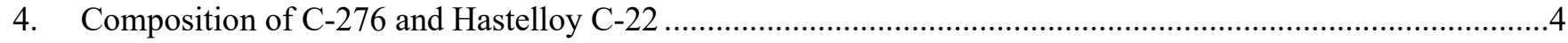

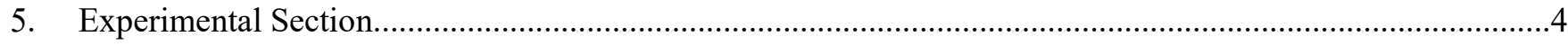

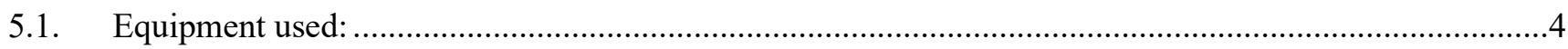

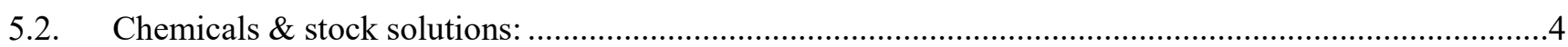

5.3. Additional C-276 coupon corrosion tests with lot B Selectfluor under various conditions. .....................5

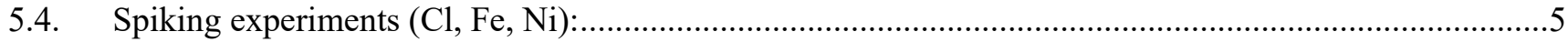

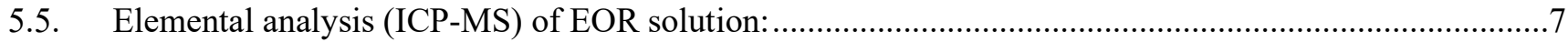

5.6. Use ${ }^{1} \mathrm{H}$ qNMR for determination of Selectfluor decomposition..............................................................

5.7. Stability of Selectfluor at different $\mathrm{Cl}^{-}$level (NMR study) ..............................................................

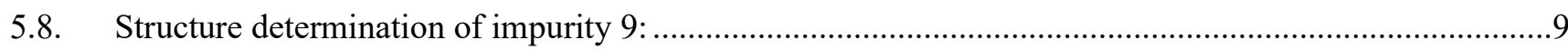




\section{General Considerations}

All reactions were carried out under an inert nitrogen atmosphere unless otherwise specified. The reagents were used as received from the commercial sources without any additional purification and drying. The 1:1 v/v $\mathrm{MeCN} / \mathrm{MeOH}$ mixture was prepared before setting up the reactions and stored over $3 \AA$ molecular sieves to keep dry. NMR spectra were acquired on $500 \mathrm{MHz}$ Bruker AVANCE III HD spectrometer equipped with inverse Prodigy probe. The chemical shifts in the ${ }^{1} \mathrm{H}$ NMR and ${ }^{13} \mathrm{C}$ NMR spectra are reported in ppm from tetramethylsilane with the solvent resonance as the internal standard.

\section{Analytical method for fluorination reaction monitoring}

Table S1. Method conditions:

\begin{tabular}{|c|c|c|c|}
\hline Column & \multicolumn{3}{|c|}{ Acquity UPLC HSS T3 2.1 x 100 mm, 1.8 um } \\
\hline Oven & \multicolumn{3}{|l|}{$40^{\circ} \mathrm{C}$} \\
\hline Autosampler Temperature & \multicolumn{3}{|l|}{ Off } \\
\hline \multirow{2}{*}{ Mobile Phases } & \multicolumn{3}{|c|}{ A: $0.1 \% \mathrm{H}_{3} \mathrm{PO}_{4}$ in $\mathrm{H}_{2} \mathrm{O}(\mathrm{v} / \mathrm{v})$} \\
\hline & \multicolumn{3}{|l|}{$\mathrm{B}: \mathrm{MeCN}$} \\
\hline \multirow{7}{*}{$\begin{array}{l}\text { Mobile phase } \\
\text { Gradient program }\end{array}$} & Time (min) & $\mathrm{A} \%$ & $\mathrm{~B} \%$ \\
\hline & Initial & 85 & 15 \\
\hline & 6 & 50 & 50 \\
\hline & 8 & 10 & 90 \\
\hline & 9 & 10 & 90 \\
\hline & 9.1 & 85 & 15 \\
\hline & 10 & 85 & 15 \\
\hline Run time & \multicolumn{3}{|c|}{$\begin{array}{l}10.00 \text { min } \\
+3 \text { min column re-equilibration time }\end{array}$} \\
\hline Flow rate & \multicolumn{3}{|l|}{$0.5 \mathrm{~mL} / \mathrm{min}$} \\
\hline Detector & \multicolumn{3}{|c|}{$210 \mathrm{~nm}$ and $233 \mathrm{~nm}$} \\
\hline Diluent & \multicolumn{3}{|c|}{$\mathrm{MeCN}: \mathrm{H}_{2} \mathrm{O} 3: 1$} \\
\hline Sample concentration & \multicolumn{3}{|l|}{$1 \mathrm{mg} / \mathrm{mL}$} \\
\hline Needle Wash: & \multicolumn{3}{|l|}{$\mathrm{MeCN}$} \\
\hline Injection volume & \multicolumn{3}{|l|}{$2.0 \mu \mathrm{L}$} \\
\hline
\end{tabular}




\section{Analytical method for $\mathrm{Cl}^{-}$and $\mathrm{F}^{-}$level measurement}

Table S2. Method conditions:

\begin{tabular}{|c|c|c|}
\hline Column & \multicolumn{2}{|c|}{ Dionex IonPac AS19 2x250 mm } \\
\hline Column Temperature: & \multicolumn{2}{|l|}{$30^{\circ} \mathrm{C}$} \\
\hline Suppressor: & \multicolumn{2}{|l|}{ AERS_2mm } \\
\hline Conductivity Cell Temp: & \multicolumn{2}{|l|}{$35^{\circ} \mathrm{C}$} \\
\hline Suppressor Current: & \multicolumn{2}{|l|}{$40 \mathrm{~mA}$} \\
\hline Eluent: & \multicolumn{2}{|c|}{ Dionex EGC $500 \mathrm{KOH}$} \\
\hline CR-TC: & \multicolumn{2}{|l|}{ On } \\
\hline Flow rate: & \multicolumn{2}{|l|}{$0.4 \mathrm{~mL} / \mathrm{min}$} \\
\hline Mobile Phase: & \multicolumn{2}{|l|}{ Water } \\
\hline \multirow{6}{*}{$\begin{array}{l}\text { Mobile phase } \\
\text { Gradient program }\end{array}$} & Time $(\mathrm{min})$ & KOH Concentration (mM) \\
\hline & Initial & 2 \\
\hline & 5 & 2 \\
\hline & 14 & 50 \\
\hline & 16 & 50 \\
\hline & 17 & 2 \\
\hline \multirow{2}{*}{ Run time } & \multicolumn{2}{|l|}{$17.00 \mathrm{~min}$} \\
\hline & \multicolumn{2}{|c|}{+5 min column re-equilibration time } \\
\hline Diluent & \multicolumn{2}{|c|}{$\mathrm{MeCN}: \mathrm{H}_{2} \mathrm{O} 1: 1$} \\
\hline Sample concentration & \multicolumn{2}{|c|}{$2 \mathrm{mg} / \mathrm{mL}$, prepared by dilution into $50 / 50 \mathrm{v} / \mathrm{v} \mathrm{MeCN} /$ water } \\
\hline Injection volume & \multicolumn{2}{|l|}{$2.0 \mu \mathrm{L}$} \\
\hline
\end{tabular}

Table S3. Fluoride and chloride content of various commercial Selectfluor batches

\begin{tabular}{|c|c|c|}
\hline Content & Fluoride Content (ppm) & Chloride content (ppm) \\
\hline Strem Lot\# 36232100 & $48634(4.9 \%)$ & $694(0.07 \%)$ \\
\hline Aldrich Lot\# STBF0638V & $53178(5.3 \%)$ & $950(0.10 \%)$ \\
\hline $\begin{array}{c}\text { Aldrich Lot\# MKCL3773 } \\
\text { (Lot A) }\end{array}$ & $47613(4.8 \%)$ & $673(0.07 \%)$ \\
\hline $\begin{array}{c}\text { PHT Chemicals Lot\# 20200101 } \\
\text { (Lot B) }\end{array}$ & $48781(4.9 \%)$ & $2610(0.26 \%)$ \\
\hline
\end{tabular}

In our process development, we tested a total of 4 lots of commercial Selectfluor (two from Aldrich, one from Strem and one from PHT Chemicals, see Table S3). We found that only 1 out of the 4 lots 
contains higher than normal $\mathrm{Cl}^{-}$content (2610 vs. $<1000$ ppm). However, we do observe chloride level variation (up to $277 \mathrm{ppm}$ ) even between two batches of Selectfluor produced by the same vendor. Therefore, as part of our control strategy, we elected to always test the $\mathrm{Cl}^{-}$content prior to use for the fluorination process.

\section{Composition of C-276 and Hastelloy C-22}

\section{Table S4. Composition of Composition of C-276 and Hastelloy C-22}

\begin{tabular}{|c|c|c|}
\hline \multicolumn{3}{|c|}{ Nominal Composition } \\
\hline & C-276 & C-22 \\
\hline Nickel & 57 (bal) & $56(\mathrm{bal})$ \\
\hline Cobalt & $2.5 \mathrm{max}$ & $2.5 \mathrm{max}$ \\
\hline Chromium & 16 & 22 \\
\hline Molybdenum & 16 & 13 \\
\hline Iron & 5 & 3 \\
\hline Tungsten & 4 & 3 \\
\hline Manganese & $1 \mathrm{max}$ & $0.5 \max$ \\
\hline Vanadium & $0.35 \mathrm{max}$ & $0.35 \mathrm{max}$ \\
\hline Silicon & $0.08 \mathrm{max}$ & $0.08 \max$ \\
\hline Carbon & $0.01 \mathrm{max}$ & $0.01 \mathrm{max}$ \\
\hline Copper & $0.5 \mathrm{max}$ & $0.5 \max$ \\
\hline
\end{tabular}

\section{Experimental Section}

\subsection{Equipment used:}

National B7990-6 40 mL. CLR vial, open top, 10/90 Septa.

IKA RET basic Stirrer Hot Plate - Safety control w/ thermocouple model RET B S001

Aluminum block pie holder - Chemglass part\# CG-1991-P-05

Aluminum Pie blocks - Chemglass part\# CG-1991-P-11, -10, -12

Magnetic Stir bars - Chemglass stir bar, magnetic X-shaped, PTFE 20x8: diameter $\mathrm{x}$ height (mm) part\# CHMGLS-CG-2013-02

C-276 coupons - Metal Samples -p/n C0400A20504000 Heat AP829 coupons $25.47 \times 12.6 \times 1.85 \mathrm{~L} \times$ $\mathrm{W} \times \mathrm{T}(\mathrm{mm})$ avg.

Branson 2800 ultrasonic bath

\subsection{Chemicals \& stock solutions:}

Lot A Selectfluor Aldrich Lot-MKCL3773

Lot B Selectfluor purchased from PHT Chemicals Lot 20200101

$3 \AA ̊$ molecular sieves: Supelco, MX1583D-1, Lot 21E0456653

MeCN/MeOH 1:1 v/v: Fisher L-206958/ Fisher L-204513, stored over 3Å molecular sieves at rt. Methanesulfonic acid Sigma Aldrich MKCL4760 
$\mathrm{Cl}^{-}$spiking solution: Dissolve 1-(chloromethyl)-1,4-diazabicyclo[2.2.2]octan-1-ium chloride (181 $\mathrm{mg}, 0.891 \mathrm{mmol}), 1: 1 \mathrm{v} / \mathrm{v} \mathrm{MeCN} / \mathrm{MeOH}(200 \mathrm{~mL}, 157.8 \mathrm{~g}) .(200 \mathrm{ppm} \mathrm{Cl}$ in solution). $\mathrm{KF}=120$ ppm.

$\mathrm{Cl}^{-}$spiking solution: Dissolve 1-(chloromethyl)-1,4-diazabicyclo[2.2.2]octan-1-ium chloride (452 $\mathrm{mg}, 2.226 \mathrm{mmol})$, in 1:1 v/v MeCN/MeOH $(200 \mathrm{~mL}, 157.8 \mathrm{~g}) .(500 \mathrm{ppm} \mathrm{Cl}$ in solution $) . \mathrm{KF}=144$ ppm.

TBA-Cl spiking solution: tetrabutyl ammonium chloride (248 mg, $0.891 \mathrm{mmol}$ ), in 1:1 v/v $\mathrm{MeCN} / \mathrm{MeOH}(200 \mathrm{~mL}, 157.8 \mathrm{~g}) .\left(200 \mathrm{ppm} \mathrm{Cl}^{-}\right.$in solution)

$\mathrm{KCl}$ spiking solution: Dissolve potassium chloride $(66.5 \mathrm{mg}, 0.891 \mathrm{mmol})$ in $1: 1 \mathrm{v} / \mathrm{v} \mathrm{MeCN} / \mathrm{MeOH}$ (200 mL, $157.8 \mathrm{~g})$. (200 ppm $\mathrm{Cl}^{-}$in solution)

Fe spiking solution: Dissolve iron(II) tetrafluoroborate hexahydrate $(49.2 \mathrm{mg}, 0.141 \mathrm{mmol})$ in $1: 1$ $\mathrm{v} / \mathrm{v} \mathrm{MeCN} / \mathrm{MeOH}$ (200 mL, $157.8 \mathrm{~g})$. (50 ppm Fe in solution) Store over $3 \AA$ molecular sieves at rt. $\mathrm{KF}=80 \mathrm{ppm}$.

Ni spiking solution: Dissolve nickel(II) tetrafluoroborate hexahydrate (458 mg, $1.344 \mathrm{mmol}$ ) in 200 $\mathrm{mL}$ of $1: 1 \mathrm{v} / \mathrm{v} \mathrm{MeCN} / \mathrm{MeOH}$. (500 ppm Ni in solution) Store over $3 \AA$ molecular sieves at $\mathrm{rt}$. KF $=$ $131 \mathrm{ppm}$.

Alconox - detergent powder

\subsection{Additional C-276 coupon corrosion tests with lot B Selectfluor under various conditions.}

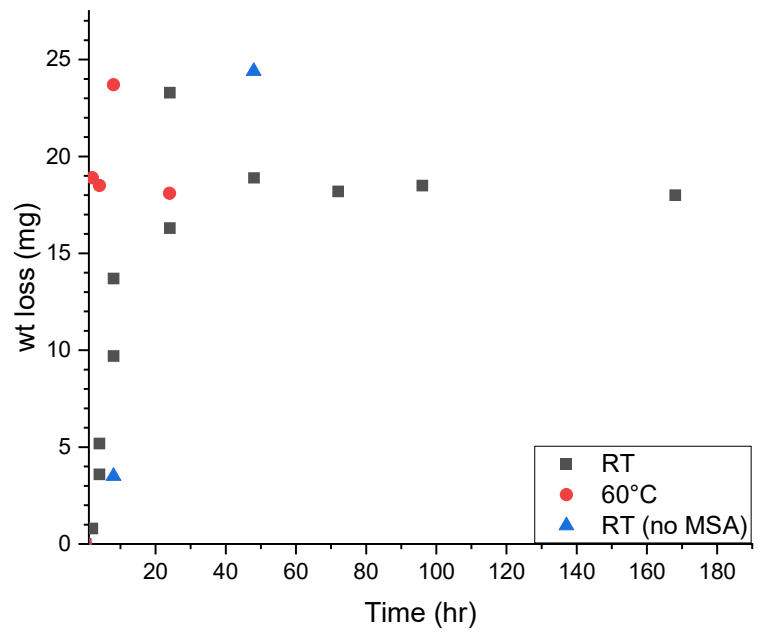

\subsection{Spiking experiments (Cl, Fe, Ni):}

For the spiking experiments, the appropriate amount of stock solution was added (see Table S5 to Table S9 below for detailed amount). Then the appropriate amount of 1:1 v/v MeCN/MeOH solution was added to bring the total solvent volume to $30 \mathrm{~mL}$, along with the Lot A or Lot B Selectfluor and Methanesulfonic acid.

Table S5. Solution $\mathrm{Cl}^{-}$content calculation*

\begin{tabular}{|c|c|c|c|}
\hline Solution & Cl- mass in solid (ug) & Total mass of solution (g) & Solution $\mathbf{C l}^{-}$content (ppm) \\
\hline Lot A Selectfluor & 673 & 24.7 & 27 \\
\hline Lot B Selectfluor & 2610 & 24.7 & 106 \\
\hline
\end{tabular}

*Calculation based on dissolving $1 \mathrm{~g}$ of Selectfluor into $30 \mathrm{~mL}$ of $1: 1 \mathrm{v} / \mathrm{v} \mathrm{MeCN} / \mathrm{MeOH}$ 
Table S6. Cl- spiking experiment

\begin{tabular}{|c|c|c|c|}
\hline Reaction & $\begin{array}{c}\text { Spike } \mathbf{C l}^{-} \text {level } \\
(\mathbf{p p m})\end{array}$ & $\begin{array}{c}\text { Vol. of pure } \\
\text { solvent (mL) }\end{array}$ & $\begin{array}{c}\text { Vol. of 200 ppm } \\
\mathbf{C l}^{-} \text {stock (mL) }\end{array}$ \\
\hline 1 & 29 & 25.67 & 4.33 \\
\hline 2 & 56 & 21.60 & 8.40 \\
\hline 3 & 83 & 17.54 & 12.46 \\
\hline 4 & 110 & 13.47 & 16.53 \\
\hline
\end{tabular}

Table S7. Reagent used/setup for Fe/Ni spiking experiments

\begin{tabular}{|c|c|c|}
\hline Cl stock & 500 & $\mathrm{ppm}$ \\
\hline Ni stock & 500 & $\mathrm{ppm}$ \\
\hline Fe stock & 50 & $\mathrm{ppm}$ \\
\hline Sel. F Cl- (Lot A) & 673 & $\mathrm{ug} / \mathrm{g}$ \\
\hline Solution mass & 24.7 & $\mathrm{~g}$ \\
\hline Solution volume & 30 & $\mathrm{~mL}$ \\
\hline Density of sol. & 0.786 & $\mathrm{~g} / \mathrm{mL}$ \\
\hline
\end{tabular}

Table S8. Fe spiking experiment setup

\begin{tabular}{|c|c|c|c|c|c|c|}
\hline $\begin{array}{c}\text { Ent } \\
\text { ry }\end{array}$ & $\begin{array}{c}\text { Spiked Fe } \\
\text { / ppm }\end{array}$ & $\begin{array}{c}\text { Spiked Cl } \\
\text { / } \mathbf{p p m}\end{array}$ & $\begin{array}{c}\text { Cl- stock solution } \\
\text { volume / mL }\end{array}$ & $\begin{array}{c}\text { Fe stock solution } \\
\text { volume / mL }\end{array}$ & $\begin{array}{c}\text { Pure 1:1 v/v } \\
\text { MeCN/MeOH Volume / } \\
\mathbf{m L}\end{array}$ & $\begin{array}{c}\text { Coupon weight } \\
\text { loss / mg }\end{array}$ \\
\hline 1 & 0 & 54 & 3.4 & 0.00 & 26.60 & 13.4 \\
\hline 2 & 5 & 54 & 3.4 & 3.14 & 23.46 & 13.2 \\
\hline 3 & 10 & 54 & 3.4 & 6.28 & 20.32 & 12.3 \\
\hline 4 & 20 & 54 & 3.4 & 12.57 & 14.03 & 12 \\
\hline 5 & 30 & 54 & 3.4 & 18.85 & 7.75 & 9.8 \\
\hline 6 & 40 & 54 & 3.4 & 25.14 & 1.46 & 15.2 \\
\hline
\end{tabular}

Table S9. Ni spiking experiment setup

\begin{tabular}{|c|c|c|c|c|c|c|}
\hline $\begin{array}{c}\text { Ent } \\
\text { ry }\end{array}$ & $\begin{array}{c}\text { Spiked Ni } \\
\text { / } \mathbf{p p m}\end{array}$ & $\begin{array}{c}\text { Spiked Cl } \\
\text { / } \mathbf{p p m}\end{array}$ & $\begin{array}{c}\text { Cl- stock solution } \\
\text { volume / } \mathbf{~ m L}\end{array}$ & $\begin{array}{c}\text { Fe stock solution } \\
\text { volume / } \mathbf{~} L\end{array}$ & $\begin{array}{c}\text { Pure 1:1 v/v } \\
\text { MeCN/MeOH Volume / } \\
\mathbf{m L}\end{array}$ & $\begin{array}{c}\text { Coupon weight } \\
\text { loss / } \mathbf{~ m g ~}\end{array}$ \\
\hline 1 & 0 & 54 & 3.4 & 0.00 & 26.60 & 14.7 \\
\hline 2 & 50 & 54 & 3.4 & 3.14 & 23.46 & 15.3 \\
\hline 3 & 100 & 54 & 3.4 & 6.28 & 20.32 & 15.1 \\
\hline 4 & 200 & 54 & 3.4 & 12.57 & 14.03 & 17.3 \\
\hline 5 & 300 & 54 & 3.4 & 18.85 & 7.75 & 17.2 \\
\hline 6 & 400 & 54 & 3.4 & 25.14 & 1.46 & 11.4 \\
\hline
\end{tabular}




\subsection{Elemental analysis (ICP-MS) of EOR solution:}

EOR solution $(50 \mathrm{uL})$ was diluted to $5 \mathrm{~mL}$ with $\mathrm{HNO}_{3}$ solution (aqueous, prepared by diluting 80 $\mathrm{mL} 63.5 \% \mathrm{w} / \mathrm{v}$ nitric acid into $100 \mathrm{~mL}$ inside of plastic volumetric flask with deionized water) and subjected to ICP-MS analysis.

\subsection{Use ${ }^{1} \mathrm{H}$ qNMR for determination of Selectfluor decomposition}

Selectfluor (3) is a strong oxidant and will decompose to $\mathbf{2}$ in presence of $\mathrm{Cl}^{-}$. To determine percent decomposition of Selectfluor $(\mathbf{2} / \mathbf{3})$, a sample $(\sim 100 \mathrm{uL})$ of reaction solution is diluted into $\mathrm{CD}_{3} \mathrm{CN}$ $(\sim 500 \mathrm{uL})$ and analyzed by ${ }^{1} \mathrm{H}$ qNMR. As shown in Figure S1, the ratio of peak e vs. $\mathbf{c}$ integration (or any combination of $\mathbf{e} / \mathbf{f}$ vs. $\mathbf{b} / \mathbf{c}$ integration) represents the ratio of $\mathbf{2} / \mathbf{3}$. Decomposition percent is defined by ratio of $\mathbf{2} /(\mathbf{2}+\mathbf{3})$. For the example shown in Figure S1, decomposition $\%=20.7 \%$.

Figure S1. Use NMR for determination of Selectfluor decomposition

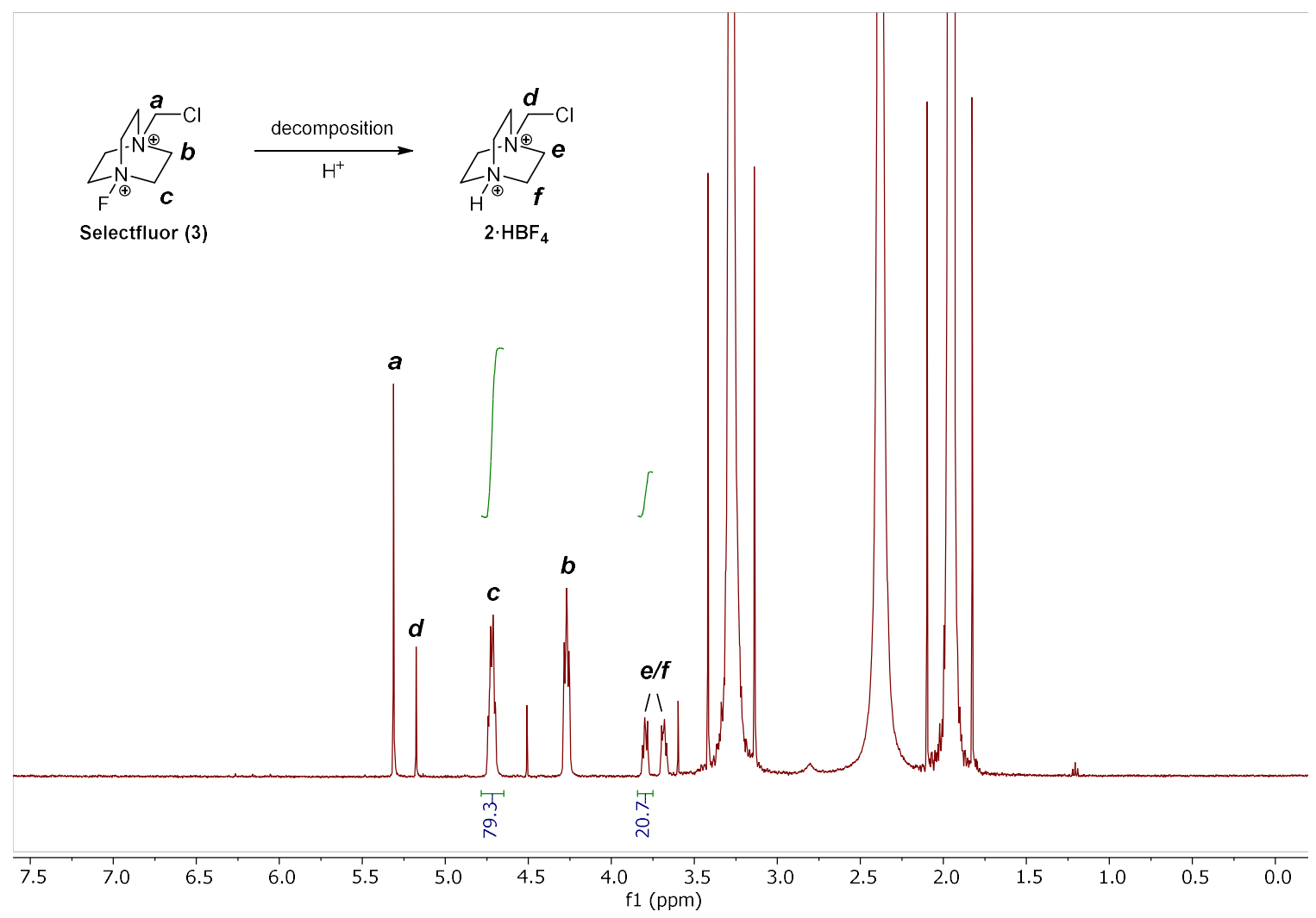

\subsection{Stability of Selectfluor at different $\mathrm{Cl}^{-}$level (NMR study)}
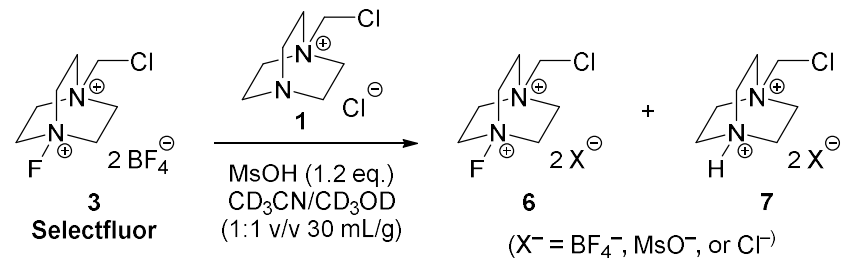

Prepare 2000 ppm stock solution of $\mathrm{Cl}^{-}$: Dissolve $48.2 \mathrm{mg}$ of compound 1 into $5 \mathrm{~mL}$ (4.33 g) of $1: 1 \mathrm{v} / \mathrm{v}$ $\mathrm{CD}_{3} \mathrm{CN} / \mathrm{CD}_{3} \mathrm{OD}$. 
Prepare 200 ppm stock solution of $\mathrm{Cl}^{-}$: Take $2 \mathrm{~g}$ of $2000 \mathrm{ppm} \mathrm{Cl}^{-}$solution prepared above and dilute into $20 \mathrm{~g}$ solution with $1: 1 \mathrm{v} / \mathrm{v} \mathrm{CD}_{3} \mathrm{CN} / \mathrm{CD}_{3} \mathrm{OD}$.

Prepare different mixtures of pure $1: 1 \mathrm{v} / \mathrm{v} \mathrm{CD}_{3} \mathrm{CN} / \mathrm{CD}_{3} \mathrm{OD}$ and $200 \mathrm{ppm} \mathrm{Cl}^{-}$stock solution according to Table S10. Charge MsOH (1.1 uL, 17 umol, 0.2 equiv) and Selectfluor (3, $30 \mathrm{mg}, 85$ umol, 1 equiv) to each reaction. After complete dissolution of Selectfluor, transfer each reaction mixture to an NMR tube. These reactions were carried out in NMR tubes directly and was heated to $60{ }^{\circ} \mathrm{C}$ in an oil bath. The percent decomposition of Selectfluor was monitored by ${ }^{1} \mathrm{H}$ qNMR according to method described above.

\section{Table S10. Ratio of pure solvent and $\mathrm{Cl}^{-}$stock solution for each reaction.}

\begin{tabular}{|c|c|c|}
\hline $\begin{array}{c}\text { Target } \mathbf{C l}^{-} \\
\text {content }\end{array}$ & $\begin{array}{c}\text { Vol. of pure solvent } \\
\text { (uL) }\end{array}$ & $\begin{array}{c}\text { Vol. of 200 ppm } \mathbf{~ C l}^{-} \\
\text {stock (uL) }\end{array}$ \\
\hline 25 & 900 & 0 \\
\hline 55 & 760 & 140 \\
\hline 81 & 638 & 262 \\
\hline 107 & 517 & 383 \\
\hline 133 & 395 & 505 \\
\hline
\end{tabular}

Figure S2. Selectfluor stability at different $\mathrm{Cl}^{-}$concentrations in absence of $\mathrm{C}-276$

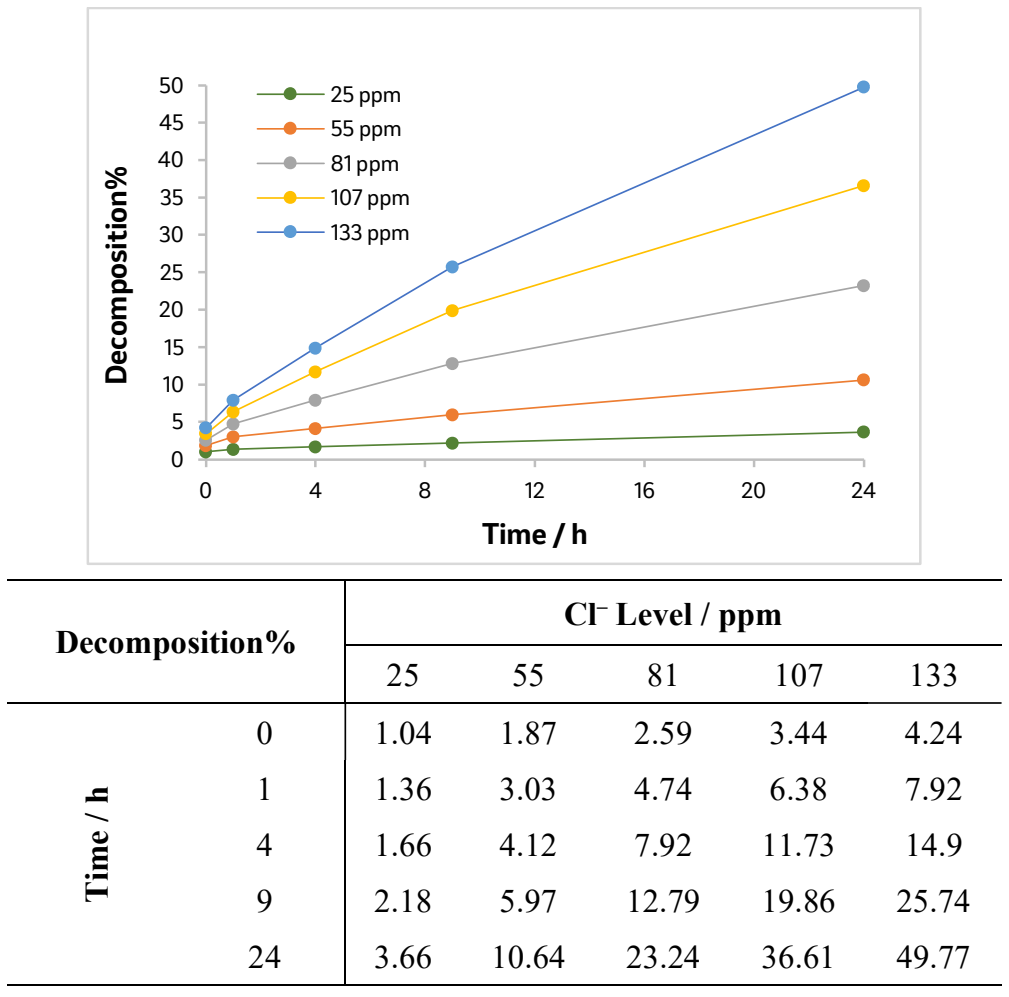




\subsection{Structure determination of impurity 9 :}

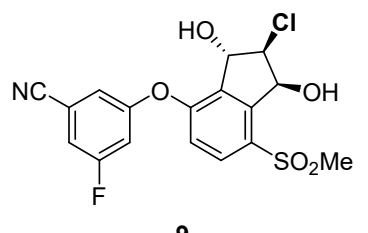

${ }^{1}$ H NMR (500 MHz, DMSO-d6): $\delta 7.89$ (dd, $\left.J=8.6,0.7 \mathrm{~Hz}, 1 \mathrm{H}\right), 7.69$ (ddd, $J=8.4,2.4,1.3 \mathrm{~Hz}$, $1 \mathrm{H}), 7.40(\mathrm{dd}, J=2.7,1.2 \mathrm{~Hz}, 1 \mathrm{H}), 7.35(\mathrm{~d}, J=10.0 \mathrm{~Hz}, 1 \mathrm{H}), 7.25(\mathrm{~d}, J=8.6 \mathrm{~Hz}, 1 \mathrm{H}), 6.07(\mathrm{~d}, J=$ $7.6 \mathrm{~Hz}, 1 \mathrm{H}), 5.96(\mathrm{~d}, J=7.5 \mathrm{~Hz}, 1 \mathrm{H}), 5.53(\mathrm{dd}, J=7.6,5.2 \mathrm{~Hz}, 1 \mathrm{H}), 5.15(\mathrm{t}, J=7.0 \mathrm{~Hz}, 1 \mathrm{H}), 4.34$ (dd, $J=6.6,5.3 \mathrm{~Hz}, 1 \mathrm{H}), 3.32(\mathrm{~s}, 3 \mathrm{H})$

${ }^{13}$ C NMR (126 MHz, DMSO-d6): $\delta$ 161.5, 157.8 (d, J = 12.0 Hz), 155.7, 143.4, 135.3, 133.4, 131.7, 120.7, $118.5(\mathrm{~d}, \mathrm{~J}=3.4 \mathrm{~Hz}), 117.2,114.8(\mathrm{~d}, \mathrm{~J}=25.5 \mathrm{~Hz}), 113.6(\mathrm{~d}, \mathrm{~J}=12.3 \mathrm{~Hz}), 111.7$ (d, J = 25.1 $\mathrm{Hz}), 77.2,70.3,68.7,44.9$.

HMRS (ESI-): Calcd. For [ $\left.\mathrm{C}_{17} \mathrm{H}_{12} \mathrm{ClFNO}_{5} \mathrm{~S}^{-}\right]\left(\mathrm{M}-\mathrm{H}^{+}\right), 396.0114$; Found, 396.0132. 


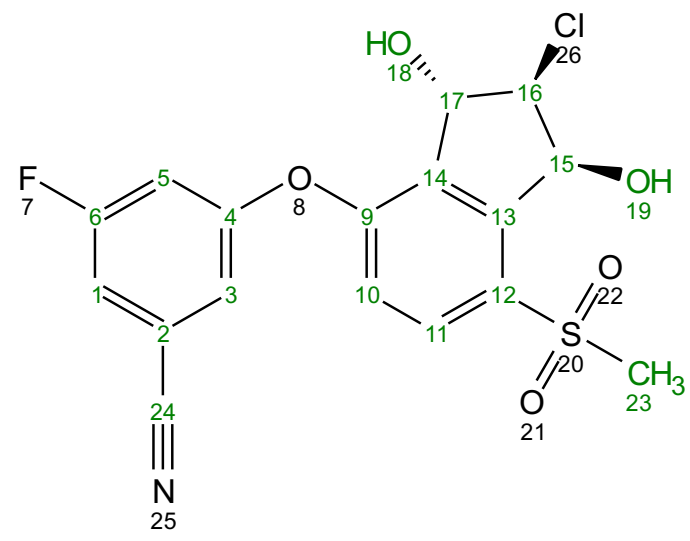

overlapped with water

5

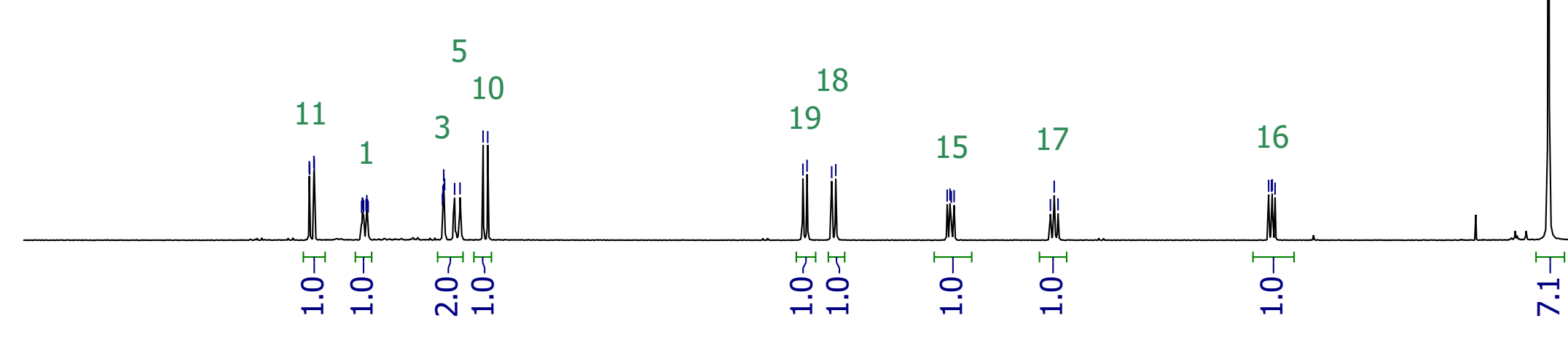


워요

ตึ่

可浆
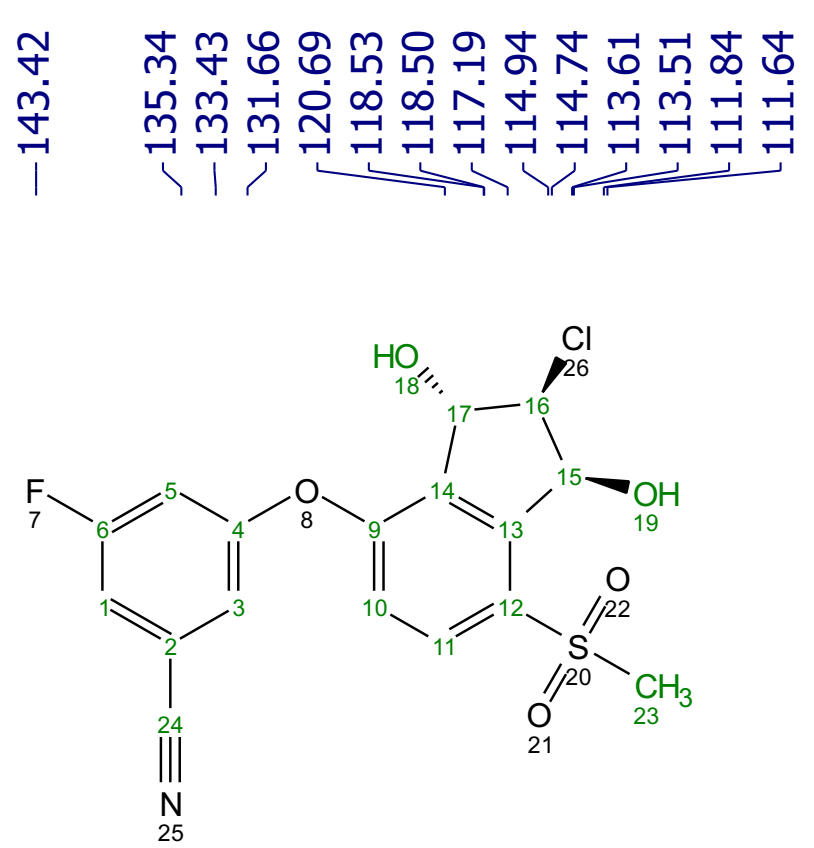
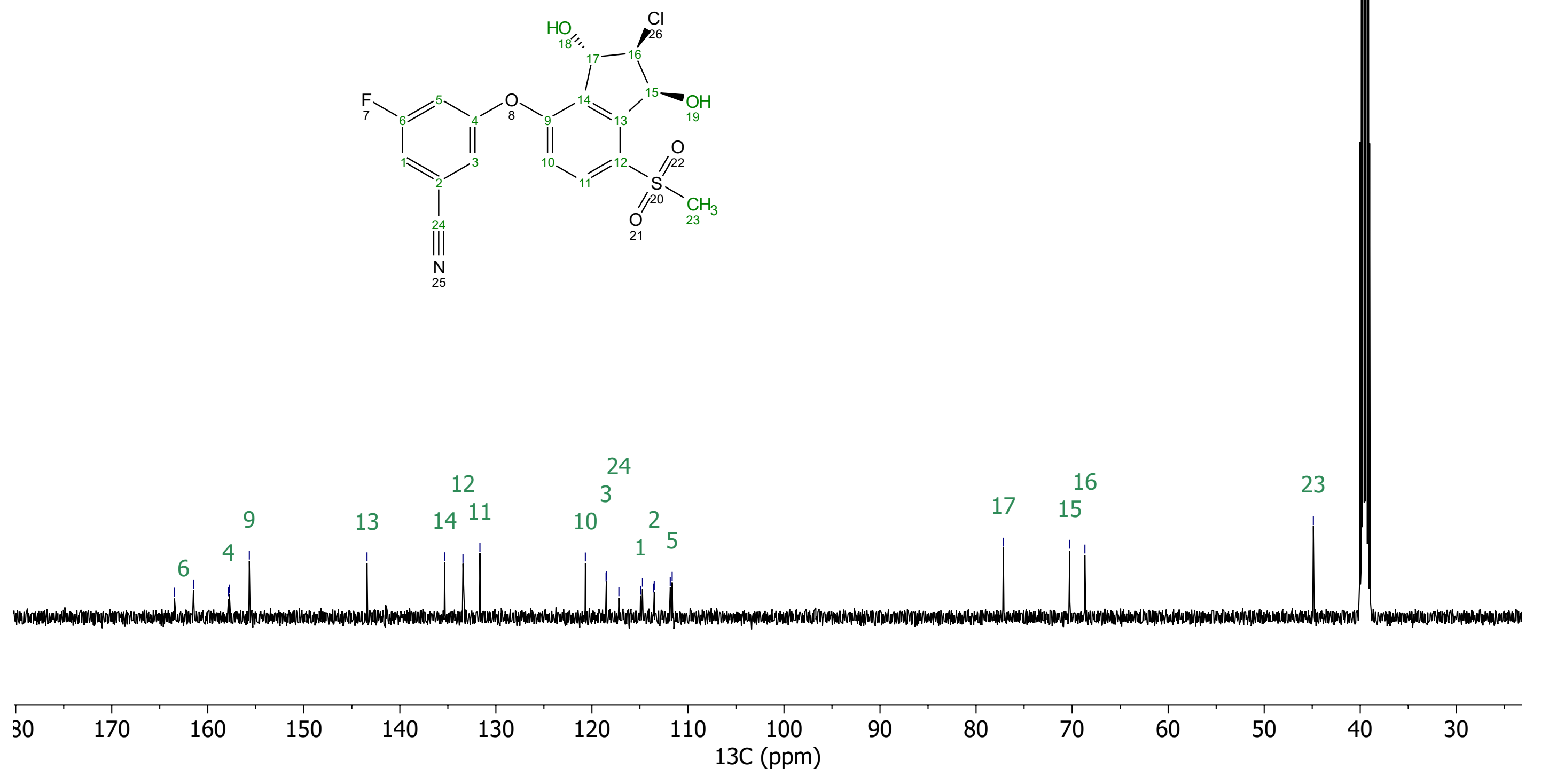